\title{
A Static Voltage Stability Index base on the Network Admittance Matrix
}

\author{
Shuyu ZHANG ${ }^{1, a}$, Qiang GUO ${ }^{1, b}$, Qinyong ZHOU ${ }^{1, c}$ \\ ${ }^{1}$ China Electric Power Research Institute, Haidian District, Beijing 100192, China \\ azhangsy920616@163.com, \\ bguoqiang@epri.sgcc.com.cn, \\ 'qyzhou@epri.sgcc.com.cn
}

\begin{abstract}
Keywords: static voltage stability; voltage stability index; network admittance matrix; voltage weak area

Abstract. A static voltage stability index base on the network admittance matrix is proposed in this paper, in order to measure static voltage stability at each bus. Compared with the existing analysis indexes, this index has the advantages of fast calculation, wide applicability and high accuracy. In this paper, the performance of the IEEE-39 system is simulated and analyzed, and the static voltage stability of Beijing power grid is studied by using the index. The results show that the index can well reflect the bus voltage stability under various operating conditions, and can be used to determine the system's weak voltage area by comparing the index values of each bus.
\end{abstract}

\section{Introduction}

A variety of static analysis indicators of voltage stability have been proposed so far, which can be divided into two categories: state index and margin index. The state indexes include the sensitivity index, the characteristic value index, the index based on the power flow solution, the local index, the impedance modulus margin index and so on[1,2,3,4,5,6]. With the help of the stability index, the network operator can identify the weak areas in the power system, so as to determine the risk of voltage instability. Through the identification of the weak link of the system, some reactive power compensation can be used to improve the voltage stability of the key bus or region.

At present, among many static indicators used in the project, the impedance modulus margin calculation has high computational efficiency, which can be used to determine the heavy load buses and the key weak areas online. But the index is used to describe the nonlinear process of linear index system problems, so it is not accurate enough, and it is difficult to obtain the Thevenin equivalent parameters of the system, which restricts the development of the method[3,4]. The sensitivity method is used to analyze the stability of the system by calculating the sensitivity of the system variables to the disturbance. This method can give the index of voltage stability, and can easily identify the strengths and weaknesses of each bus in the system, and give the corresponding countermeasures. The most common sensitivity criteria are $\mathrm{dU} / \mathrm{dQ}$, dU/dP, etc. But the sensitivity index can only consider the influence of a single variable on the stability of the system, which will bring large error, and when the system is overloaded and the generator's reactive power reaches the limit, the bus will change from the PV bus to the PQ bus, leading to sharp changes in sensitivity[5,6].

In this paper, a static voltage stability index based on the admittance matrix, which is called voltage interrelated margin index(VIMI), is derived according to the basic power flow formula. The index value can be calculated, when the voltage phase and network admittance matrix of each bus in the system is known. The index can be used to judge the voltage stability of each bus and the weak area of the system voltage, and can take into account the influence of the load of the connected bus on the voltage stability of a certain bus. Compared with the existing static voltage stability analysis index, the index has the advantages of fast calculation, wide applicability and high accuracy.

\section{Voltage Interrelated Margin Index}

Theoretical Derivation of the Index. When the load of the system reaches the transmission power limit, the system will be in a critical state. In this state, if the load increases a certain amount, the 
system voltage level will drop rapidly, leading to voltage instability. In static voltage stability analysis, usually the critical power flow solution is treated as the voltage stability limit, which value is corresponding to the singular point of Jacobian matrix of power flow equations.

For a N-bus system, the complex power of at bus $k$ can be written as:

$$
S_{k}^{*}=\left|V_{k}\right|^{2} \sum_{\substack{m=1 \\ m \neq k}}^{N} Y_{k m}-V_{k}^{*} \sum_{\substack{m=1 \\ m \neq k}}^{N} V_{m} Y_{k m}
$$

Replace the mutual admittance by self admittance, then type (1) is equivalent to:

$$
\frac{S_{k}^{*}}{Y_{k k}}=\left|V_{k}\right|^{2}-\left(\left|V_{k}\right| \cos \delta_{k}-j\left|V_{k}\right| \sin \delta_{k}\right) * \sum_{\substack{m=1 \\ m \neq k}}^{N}\left(\left|V_{m}^{\prime}\right| \cos \delta_{m}^{\prime}+j\left|V_{m}^{\prime}\right| \sin \delta_{m}^{\prime}\right)
$$

The $V_{m}{ }^{\prime}$ in (2) is given by:

$$
V_{m}^{\prime}=\frac{Y_{k m}}{\sum_{\substack{j=1 \\ j \neq k}}^{N} Y_{k j}} V_{m}
$$

where $V_{k}$ is the voltage phase at bus $k, V_{m}$ is the voltage phase at bus $m, \delta_{k}$ is the voltage angle of bus $k, Y_{k m}$ is the admittance between buses $k$ and $m$.

The right-hand side of (2) is a complex quantity, which is of the form $a-j b$. The following two equations with two unknowns $\left(V_{k}, \delta\right)$ can be written as:

$$
\begin{aligned}
& f_{1}\left(V_{k}, \delta\right)=\left|V_{k}\right|^{2}-\sum_{\substack{m=1 \\
m \neq k}}^{N}\left|V_{m}^{\prime}\right|\left|V_{k}\right| \cos \delta \\
& f_{2}\left(V_{k}, \delta\right)=\sum_{\substack{m=1 \\
m \neq k}}^{N}\left|V_{m}^{\prime}\right|\left|V_{k}\right| \sin \delta
\end{aligned}
$$

Solving the two equations of (4) and (5) using the Newton-Raphson technique. When the Jacobian matrix is singular, the corresponding solutions are at the critical stable state. And there has:

$$
2\left|V_{k}\right| \cos \delta=\sum_{\substack{m=1 \\ m \neq k}}^{N}\left|V_{m}^{\prime}\right|
$$

Equation(6) using complex number identities can be written as:

$$
\left|1-\frac{\sum_{\substack{m=1 \\ m \neq k}}^{N} V_{m}^{\prime}}{V_{k}}\right|=1
$$

The voltage interrelated margin index (VIMI) of bus $k$ can be defined as follows:

$$
\text { VIMI }_{k}=\left|1-\frac{\sum_{\substack{m=1 \\ m \neq k}}^{N} V_{m}^{\prime}}{V_{k}}\right|
$$

Range and Boundary of the Index. When the VIMI value of a bus is 1, it shows that the bus is in a critical stable state, and when the VIMI is 0 , it indicates that the bus is in no-load condition. So the range of VIMI is $0 \sim 1$, and the larger the value is, the closer the bus is to the critical stable state, which means voltage stability is worse.

Advantage of the Index. Compared with the existing static voltage stability analysis index, the index VIMI has these following advantages:

1)Fast calculation: the index only needs the network admittance matrix and the voltage phase of each bus in the system. In the static stability analysis, network admittance matrix of most of the time 
will not change, and the matrix is highly sparse matrix; in addition, the equivalent calculation process is not required, nor need the inverse matrix, which can improve the calculation speed greatly.

2)Wide applicability: the use of the index don't be limited by the scale and operation mode of the system, and the physical meaning of the index at critical points is clear. Therefore, the voltage stability of different load buses and the different operating modes in the same operating mode is easy to compare, so the index has wide applicability.

3)High accuracy: all the required parameters can be obtained accurately, without idealization or equivalent linearization, and the value of the index depends on not only the bus itself, but also the influence of buses connected. So the index can more accurately reflect the static voltage stability.

\section{Simulation Examples}

In this paper, the performance of the index in analyzing the static voltage stability of the IEEE-39 system and the Beijing power grid are simulated. Due to space limitations, only partial results are listed below. The PSD-BPA tools developed by China Electric Power Research Institute are used to simulate.

Simulation Example of the IEEE-39 System. The IEEE-39 test system includes a total of 10 generators, 39 buses and 46 lines, of which bus 31 is chosen as the balance bus. The power benchmark is $100 \mathrm{MVA}$, and voltage benchmark is $100 \mathrm{kV}$. Bus 26 is one of the most important load buses in the system, which are connected with bus $25,27,28$, and 29 through the tie line. The initial active load of bus 26 is $1.4 \mathrm{pu}$, and the reactive load is $0.2 \mathrm{pu}$. Increased the reactive power of bus 26 gradually, and calculated the power flow once every $2 \mathrm{pu}$, then calculated the VIMI value of each bus according to formula (8). Reactive power against voltage and VIMI at bus 26 is shown in Fig.1.

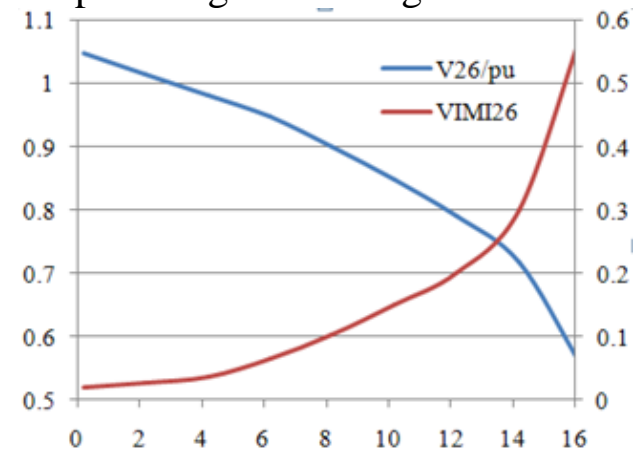

Fig.1 Reactive power at bus 26 against voltage and VIMI at bus 26

When the reactive power of bus 26 is small, the voltage of the bus is normal, and the VIMI is no more than 0.1 , but when the load increases to $16.2 \mathrm{pu}$, the voltage level of the bus is down to $0.56 \mathrm{pu}$, and the VIMI value has risen to 0.58 , which means the bus is almost reach the critical stable state. When a bus is in the critical state of stability, VIMI value of the bus is close to the critical value of 1 , so the voltage stability of a bus can be estimated by calculating the value of VIMI nodes.

At the same time, the increase of reactive power of bus 26 will cause the decrease of the voltage amplitude and the change of VIMI in other nodes connected. Reactive power at bus 26 against VIMIs at bus 25, 27, 28 and 29 are shown in Fig. 2, showing that the values of these buses also rise. The above analysis shows that when the VIMI index is used to evaluate the static voltage stability, the influence of the adjacent buses can be considered. 


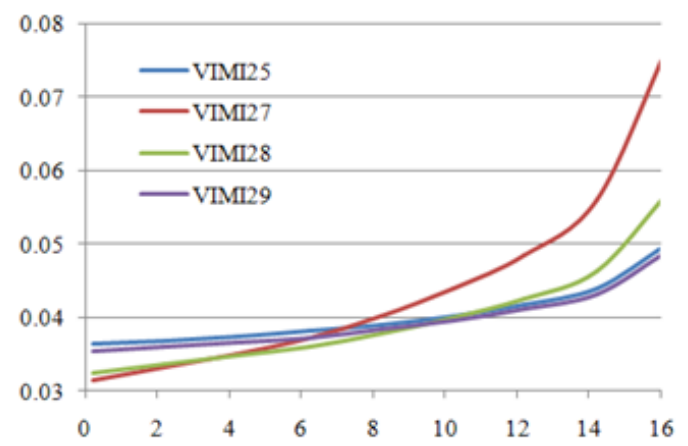

Fig.2 Reactive power at bus 26 against VIMIs at bus 25, 27, 28 and 29

Simulation Example of Beijing Power Grid. As a ultra large city power grid, Beijing power grid is an important part of Beijing-Tianjin-Hebei Power Grid. In addition to assume the task of supplying power to the capital, it also transfer power to the neighboring area. In 2017, Beijing power grid can be divided into 8 partitions, naming from A1 to A8. The periphery of the power grid is a double loop network structure formed by the $500 \mathrm{kV}$ hub substations and some south Hebei $500 \mathrm{kV}$ substations, and the interior consists four $500 \mathrm{kV}$ load stations that supply power to the city.

Based on the data of Beijing 2017 power grid, obtained the voltage amplitude and phase angle of each $220 \mathrm{kV}$ load bus, calculated the network admittance matrix and calculated the VIMIs according to formula(8), then sorted in order from large to small. The top 20 of the buses and the corresponding VIMIs are shown in table.1.

Table.1 Beijing 2017 VIMIs

\begin{tabular}{|c|c|c|c|c|c|c|c|}
\hline Number & Station & Partition & VIMI & Number & Station & Partition & VIMI \\
\hline 1 & Caoqiao & A5 & 0.2498 & 11 & Yuanda & A5 & 0.1678 \\
\hline 2 & Caishikou & A5 & 0.2435 & 12 & Haidian & A7 & 0.1668 \\
\hline 3 & Liyuan & A8 & 0.2340 & 13 & Lucheng & A5 & 0.1666 \\
\hline 4 & Yuquan & A5 & 0.2179 & 14 & Xiazhuang & A8 & 0.1639 \\
\hline 5 & Zuoanmen & A5 & 0.2117 & 15 & Yonglu & A2 & 0.1620 \\
\hline 6 & Tuanhe & A5 & 0.1889 & 16 & Chaoyang & A2 & 0.1618 \\
\hline 7 & Xingdu & A5 & 0.1875 & 17 & Yongding & A6 & 0.1548 \\
\hline 8 & Lianhua & A8 & 0.1803 & 18 & Yuege & A8 & 0.1521 \\
\hline 9 & Nanyuan & A8 & 0.1748 & 19 & Yiqian & A5 & 0.1498 \\
\hline 10 & Taoyuan & A6 & 0.1721 & 20 & Xinxi & A8 & 0.1678 \\
\hline
\end{tabular}

According to the results in Tab.1 and the corresponding geographic map, it can be found that the weak voltage areas of Beijing power grid are mainly concentrated in A5 and A8, which is consistent with the results of other indexes and the actual operation.

\section{Conclusions}

A static voltage stability index base on the network admittance matrix is proposed in this paper. The index can be used to evaluate the static voltage stability of each bus by giving the voltage phase and the admittance matrix. In this paper, the performance of the index in analyzing the static voltage stability of the IEEE-39 system and the Beijing power grid are simulated. The results show that the index can accurately reflect the bus voltage stability of a variety of operating conditions, and to determine the weak areas of the system in static voltage stability. Compared with the existing analysis indexes, this index has the advantages of fast calculation, wide applicability and high accuracy, and has certain engineering application value.

\section{References}

[1] Y. Tang: Power System Voltage Stability Analysis(Science Press, China 2011)

[2] Y. Tang, H. Sun, J. Yi: Chinese Journal of Electrical Engineering Vol. 13(2009), p. 48-53. 
[3] Y. Tang, W. Lin, H. Sun: Chinese Journal of Electrical Engineering Vol. 16(2010), p. 12-18.

[4] S. Lee, Y. Cao, Y. Liu: Chinese Journal of Electrical Engineering Vol. 22(2014), p. 91-98.

[5] J. Yuan, X. Duan: Power System Automation Vol. 4(1997), p. 7-10.

[6] Gao B, Morison K. Power Systems IEEE Transactions on, Vol. 4(1992), p. 1529-1542. 